\title{
Formation of Poly(vinyl alcohol)-Iodine Complex in Water Swollen Films on Extension
}

\author{
Yushi OISHI and Keizo MIYASAKA \\ Department of Textile and Polymeric Materials, \\ Tokyo Institute of Technology, \\ Ookayama, Meguro-ku, Tokyo 152, Japan
}

(Received July 31, 1986)

\begin{abstract}
Extension induced iodine-poly(vinyl alcohol) (PVA) complexation was studied for PVA films with different degrees of hydration in very dilute iodine aqueous solutions. The complexation was reversibly induced by extension and its recovery. The strain energy required for the complexation was estimated to understand complexation enhancement by extension. The strain energy increased with the increase of temperature, and with the decrease of the iodine concentration, while it was almost independent of the degree of hydration. This extension induced complexation is considered to be due to the decrease in the entropy of PVA chains by extension.

KEY WORDS Poly(vinyl alcohol) / Water Swollen Film / Iodine / IodinePoly(vinyl alcohol) Complex / Degree of Hydration / Extension / Strain Energy / Entropy / Dilute Solution /
\end{abstract}

Formation of the iodine-PVA complex in an aqueous solution is affected by many factors. For example, the complexation is enhanced by (I) the increase in the concentrations of PVA and iodine, ${ }^{1}$ (II) the increase in syndiotacticity of PVA, ${ }^{2}$ (III) additions of boric acid $^{3}$ and of orthotelluric acid $^{4}$ (IV) decreasing temperature, ${ }^{1,5}$ and (V) acetalization of PVA. ${ }^{6}$ The complexation does not occur at a great dilution of PVA or iodine. ${ }^{3,5,7}$ Acids, the decreasing temperature, the acetalization of PVA and the syndiotacticity of PVA seem to decrease the mobility of PVA chain or cause a chain conformation suitable for complexation, resulting in the formation of the complex.

Iodine-PVA complexation also occurs in the swollen gel state of PVA. The complexation in swollen films has been studied as functions of the iodine concentration and added amounts of boric acid ${ }^{8}$ in the soaking solution, the degree of hydration D.H. of PVA films $^{9}$ and the extension of PVA films. ${ }^{7,10}$
The effects of iodine concentration and addition of boric acid on the complexation of swollen films were similar to those in the complexation in aqueous solutions. The increase in D.H. of PVA films increased the amount of complex formed under a given soaking condition. It was also found that the extension of PVA films remarkably enhanced the complexation: when yellow colored PVA films in which no complexation had occurred were stretched in the very diluted solution ${ }^{10}$ or in the vapor of iodine, ${ }^{8}$ they changed color to blue, showing that the complexation was induced by extension. The visible absorption maximum $\lambda_{\max }$ of the complex was found to increase with extension, indicating that the length of the polyiodine chain increased with extension. The formation of PVA-iodine complex induced by extension was supposed to be due to the excess strain energy stored in PVA chains, ${ }^{10}$ in analogy to the elevation of the melting point observed in drawing natural rubber. ${ }^{11)}$ 
In this paper, we studied quantitatively the complexation induced by extension of PVA films in very dilute iodine aqueous solutions. The amounts of complex were measured as a function of extension. The excess energy required for the complexation was also measured with particular interests in the effects of D.H. of PVA films and the iodine concentration in the soaking solutions on the complexation.

\section{EXPERIMENTAL}

Details of preparation method of water swollen PVA films were described in a previous paper. ${ }^{9}$ Films $50 \mu \mathrm{m}$ thick were cast from a $7 \mathrm{wt} \%$ aqueous PVA solution at $20^{\circ} \mathrm{C}$, followed by annealing to get different D.H.s of specimens. The D.H. was defined as the volume fraction of water in a wet PVA film at the equilibrium swollen state. Iodine and potassium iodide (Wako Pure Chemical Industries Ltd.) were used without further purification. Iodine-potassium iodide aqueous solutions with a mole ratio of $1: 2$ were prepared for soaking of PVA films. The concentration of iodine in the solution was varied from $2 \times 10^{-4}$ to $9 \times 10^{-4} \mathrm{moll}^{-1}$ in this study. The equilibrium iodine sorption by the swollen films was measured after a 24 hours soaking in each solution. For evaluation of the amount of complex, the visible ray spectra of specimens extended by using the device shown in Figure 1(a) were measured by a Shimadzu UV-240 spectrophotometer in a range from $400 \mathrm{~nm}$ to $800 \mathrm{~nm}$. The stress-strain curves of swollen PVA films were obtained at a given temperature, using a device whose schematic diagram is shown in Figure 1(b). The initial length of specimen was $2 \mathrm{~cm}$ in the stretching experiments. The cross-head speed of the extensometer was $2.3 \mathrm{~mm} \mathrm{~min}^{-1}$, and the force was detected by a strain gauge.

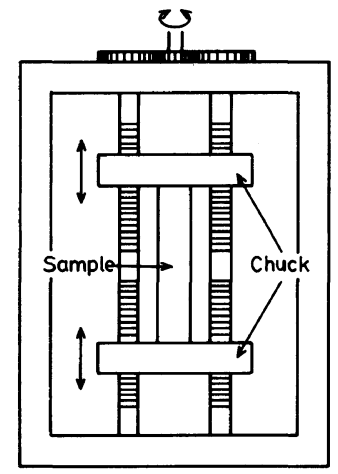

(a)

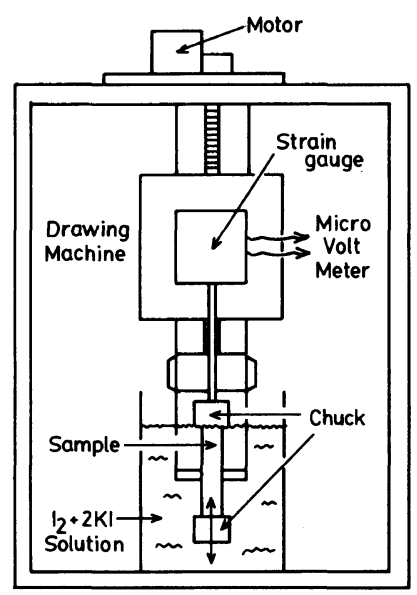

(b)

Figure 1. Schematic representation of the apparatus for (a) absorbance measurement on extension, (b) stressstrain measurement.

\section{RESULTS AND DISCUSSION}

Figure 2 shows stress-strain curves of iodine-sorbed PVA films of D.H. $=0.85,0.77$, and 0.64 , measured in solutions of 3,6 , and $9 \times 10^{-4} \mathrm{moll}^{-1}$ iodine concentrations. Prior to the stress-strain measurements, all the samples were soaked for 24 hours at the same iodine concentration of solutions at $30^{\circ} \mathrm{C}$ as those in which stress-strain curves were measured. Figure 2 shows that all the films had high extensibilities over $400 \%$ in strain with yield regions at strains of $70-120 \%$ and that the stress-strain behavior depended much on D.H. of films but not on the iodine concen- 


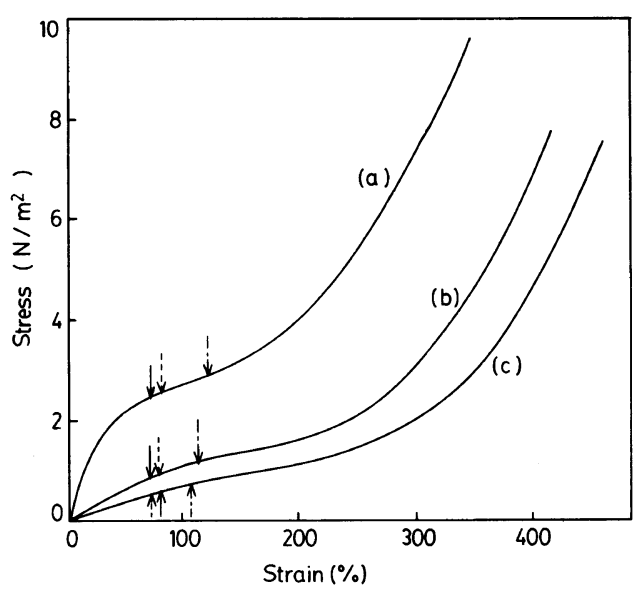

Figure 2. Stress-strain curves of iodine-sorbed PVA films of D.H.; (a) 0.64 , (b) 0.77 , (c) 0.85 at various iodine concentrations: (-- -, $3 \times 10^{-4} \mathrm{moll}^{-1}$; (----), $6 \times 10^{-4} \mathrm{moll}^{-1} ;(-), 9 \times 10^{-4} \mathrm{moll}^{-1}$ at $30^{\circ} \mathrm{C}$.

tration of the soaking solutions. The latter result may be due to the small concentration of iodine used in this study. Iodine concentrations such as would not cause any complexation were chosen (and therefore the films remained yellow in color) when the films were soaked in the solutions without extension. However, the color turned to blue during extension, indicating that the complexation was induced by extension. It should be remarked that no complexation was induced even by extension in solutions of iodine concentrations less than $2 \times 10^{-4} \mathrm{moll}^{-1}$. The arrows on the stress-strain curves indicate the point where the color of film began to change from yellow to blue, in other words, the point where the complexation began to occur. In a previous paper, ${ }^{10}$ it was shown that the stress-strain behavior was much changed by the complexation. In this case, however, it is difficult to find any apparent effect of the complexation on the stress-strain relations, which must be due to the fact that the amounts of iodine-PVA complex were very small because of the small concentration in the soaking solutions. The complexation points, on the stress-strain curves indicated by

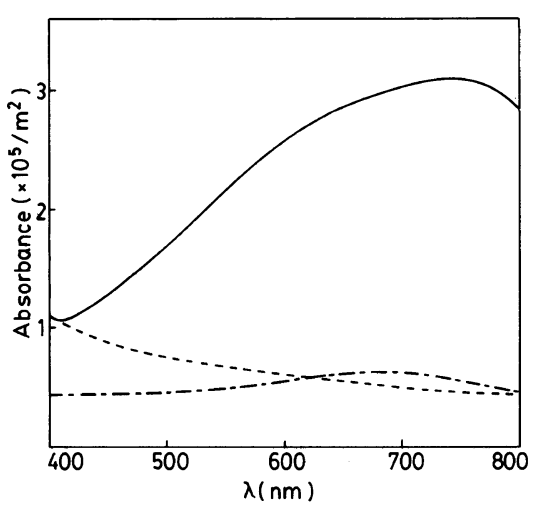

Figure 3. Absorption spectra of iodine-sorbed PVA films on extension in $3 \times 10^{-4} \mathrm{moll}^{-1}$ of iodine at $30^{\circ} \mathrm{C}$ : (-), 300\% extension in the solution; (----), $0 \%$ extension in the solution; (-- $)$, soaked in the solution after $300 \%$ extension.

arrows, appear in the yield region and shift to larger strain side with decreasing iodine concentration for a given D.H. of specimen. Next the complexation induced by extension was studied with UV-absorption using a device shown in Figure 1(a).

Figure 3 shows absorption spectra of iodine sorbed PVA films, measured before and after extension in a solution of $3 \times 10^{-4} \mathrm{~mol}$ $1^{-1}$ iodine concentration at $30^{\circ} \mathrm{C}$. Before extension, the PVA film soaked in the solution for 24 hours had no peak in the spectrum, indicating that no complexation occurred. On the contrary a broad peak appeared at about $700 \mathrm{~nm}$ when the film was extended by $300 \%$ in the solution, indicating that the complexation was induced by extension. The increase in extension of PVA films caused a shift of $\lambda_{\max }$ to longer wave length. The complexation also occurred on soaking in the dilute solutions after a $300 \%$ extension of PVA film swollen in water. These results indicate that the extended conformation of PVA chains is favorable to the complexation.

Figure 4 shows the absorbance at $\lambda_{\max }$ of a specimen of D.H. $=0.84$ soaked in the solution of $3 \times 10^{-4} \mathrm{moll}^{-1}$ iodine concentration as a function of strain at $30^{\circ} \mathrm{C}$. This absorbance is 


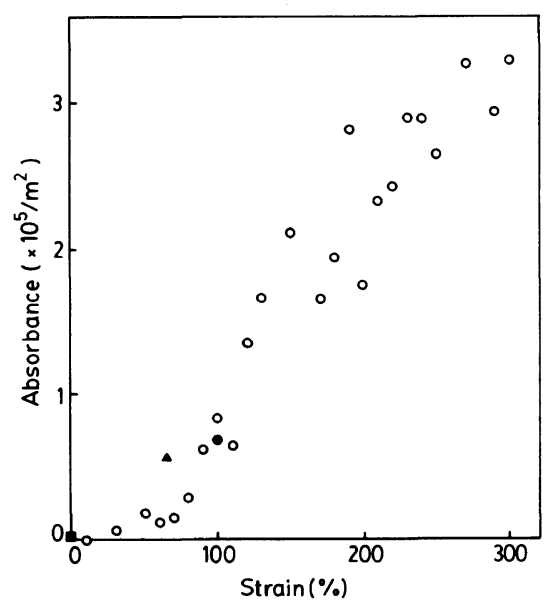

Figure 4. Absorbance as a function of strain at $30^{\circ} \mathrm{C}$, together with the absorbance on recovery: $(\mathbf{O})$, recovery from $300 \%$ extension; $(\Delta)$, recovery from $200 \%$ extension; (ם), recovery from $100 \%$ extension.

assumed to be proportional to the equilibrium amount of complex. ${ }^{9}$ The amount of complex suddenly became recognizable at the strain indicated by each arrow in Figure 2, followed by successive increase with extension. A residual strain was caused after high extension over $100 \%$ extension. The amounts of complex measured after removal of extension force are also shown by solid points in Figure 4. The abscissa for these solid points corresponds to the residual strain after removal of extension force. The results that the solid points lie among the group of open points which were measured on the course of extension and that the complexes formed by extension less than the yield points completely dissociate after removal of strains indicate that the complexation is reversibly induced by extension and its recovery. The reversible complex formation caused by extension may be qualitatively explained by the decrease in the entropy of PVA chain due to the chain extension.

Let $g_{\mathrm{c}}$ and $g_{\mathrm{s}}$ be the free energy of the complex and non-complex phases, respectively. $g_{\mathrm{s}}$ depends not only on the concentrations of iodine and PVA, and temperature but also on the conformational energy of
PVA chains related to the entropy of PVA chains. The point $T_{\mathrm{d}}$ where $g_{\mathrm{c}}$ is equal to $g_{\mathrm{s}}$ corresponds to the decomposition temperature of complex. The non-complex phase is stable above $T_{\mathrm{d}}$. At $T_{\mathrm{d}}$, the following equation must be satisfied,

$$
T_{\mathrm{d}}=\Delta H_{\text {comp }} / \Delta S_{\text {comp }}
$$

where $\Delta H_{\text {comp }}$ and $\Delta S_{\text {comp }}$ are the differences in enthalpy and entropy between complex and non-complex. $T_{\mathrm{d}}$ depends on the iodine concentration and the state of PVA chains, as $g_{\mathrm{s}}$ is a function of them under given pressure and polymer concentration (which is considered to closely related to D.H.). If $T_{\mathrm{d}}$ is lower than the soaking temperature, no complex occurs. This is the case of very low iodine concentration. Let us consider a case where PVA chains in the non-complex phase are stretched to increase the $g_{\mathrm{s}}$ mainly due to the decrease in their conformational entropy. This increase in $g_{\mathrm{s}}$ necessarily shifts $T_{\mathrm{d}}$ where $g_{\mathrm{s}}$ is equal to $g_{\mathrm{c}}$ to higher temperature. When $T_{\mathrm{d}}$ becomes equal to the soaking temperature, the complexation becomes possible. The enhancing effects of addition of acids to iodine solutions $s^{3,4,8}$ and the syndiotacticity ${ }^{2}$ and acetalization ${ }^{6}$ of PVA on the complexation may be explained also by decreased entropy of PVA chains. The experimental estimation of strain energy required for complexation must be important to understand the complexation enhancement by extension. Thus we studied the strain energy corresponding to the complexation point as functions of temperature, iodine concentration and D.H. of PVA films which depended on the fine structure.

Figure 5 shows the total strain energy (standardized by the unit volume of swollen film) corresponding to the complexation point, as a function of D.H. at different temperatures. The total energy remarkably decreases with increasing D.H. and is smaller at lower temperature.

This strain energy was standardized further by the unit volume of PVA, taking into con- 
sideration the situation that the strain energy was related to the extension of PVA components. The tendency that the strain energy decreases with the increase in D.H. was still kept in this case, though the dependency on D.H. decreased more than before. The total

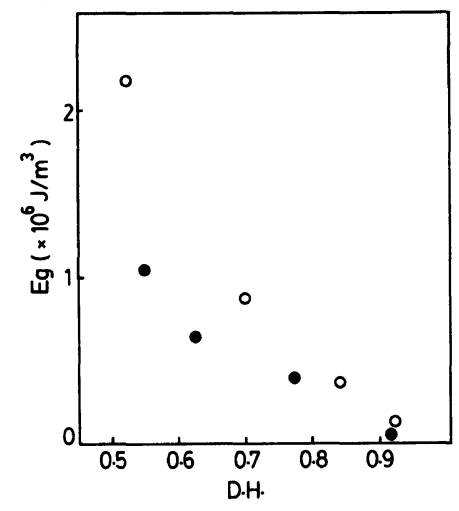

Figure 5. Total energy per unit volume of gel as a function of D.H., at $30^{\circ} \mathrm{C}(\mathrm{O})$ and at $5^{\circ} \mathrm{C}(\mathrm{O})$.

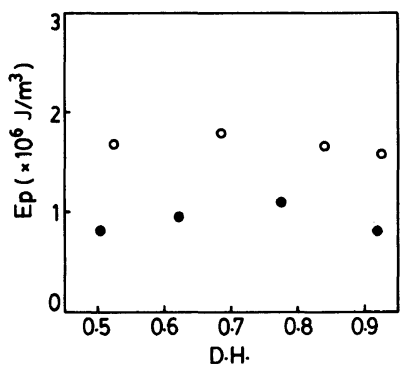

Figure 6. Strain energy on recovery per unit volume of polymer as a function of D.H., at $30^{\circ} \mathrm{C}(\mathrm{O})$ and at $5^{\circ} \mathrm{C}$ (O). strain energy is contributed not only by the energy stored in chains but also by the energy dissipated during the extension. Thus, we should take only the energy stored in chains. It is not difficult to estimate the energy stored in chains from the hysteresis curves of stressstrain, unless the extension is very high. As mentioned above, some residual extension of chains occured in highly extended specimens. The energy to cause the residual extension of chains is recognized as apparently "dissipated energy", even though it is originally "stored energy". However, this problem is not important in cases where no appreciable residual strain is caused. The stored energy was estimated from a cycle of extension to the complexation point and the recovery.

In Figure 6, the strain energy thus obtained for the complexation is shown as a function of D.H., indicating that the energy is almost independent of the D.H. of PVA films, and also that the values at $5^{\circ} \mathrm{C}$ are less than those at $30^{\circ} \mathrm{C}$ at a given iodine concentration. This is in agreement with the results shown in a previous paper $^{9}$ that the lower temperature is favorable to the complexation, i.e., the decreased temperature caused an increase in the amount of complex formed at a given iodine concentration, and also the shifts of $\lambda_{\max }$ to longer wave lengths. Because of the dependence of $T_{\mathrm{d}}$ on the iodine concentration, the strain energy required for complexation must be larger at lower than higher iodine concen-
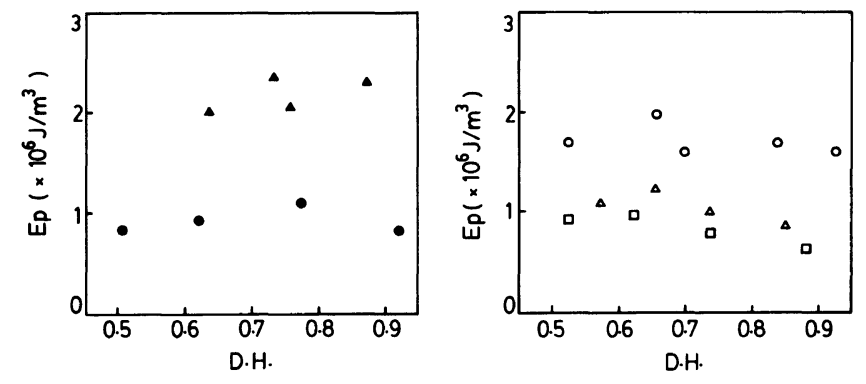

Figure 7. Dependence of the concentration of iodine on the strain energy at $5^{\circ} \mathrm{C}$ in $3 \times 10^{-4} \mathrm{moll}^{-1}(\bigcirc)$, $2 \times 10^{-4} \mathrm{moll}^{-1}(\Delta)$, LEFT; at $30^{\circ} \mathrm{C}$ in $9 \times 10^{-4} \mathrm{moll}^{-1}(\bigcirc), 6 \times 10^{-4} \mathrm{moll}^{-1}(\triangle), 3 \times 10^{-4} \mathrm{moll}^{-1}(\square)$, RIGHT. 
trations, when the iodine concentration is changed at a given temperature.

Figure 7 shows the iodine concentration dependence of the strain energy for complexation at 5 and $30^{\circ} \mathrm{C}$. The strain energy decreases with the increase of iodine concentration at both temperatures. The strain energy for complexation, however, has a very small dependence on D.H. at every iodine concentration.

Thus, the formation of PVA-iodine complex induced by extension is explained on energetics by the decrease in the entropy of PVA chains. The helical model and the extended model have been proposed for the structure of PVA-iodine complex. ${ }^{1}$ The complex formation on soaking in the dilute solution before and after extension of PVA film as shown in Figure 3 indicates that the extended conformation of PVA chains is favorable to the complexation. The remarkable increase in Young's modulus on soaking film in iodine aqueous solution indicates that extra junction points due to iodine complexes are formed. ${ }^{10}$ We now support the latter model that the iodine chain is surrounded by several extended PVA segments.

\section{REFERENCES}

1. M. M. Zwick, J. Appl. Polym. Sci., 9, 2393 (1965); L. G. Tebelev, G. F. Mikul'skii, Ye. P. Korchagina, and S. A. Glikman, Vysokomol. Soedin., Ser. A, 7, 1231 (1965).

2. K. Kikukawa, S. Nozakura, and S. Murahashi, Polym. J., 2, 212 (1971); S. Matsuzawa, K. Yamamura, and H. Noguchi, Makromol. Chem., 175, 31 (1974).

3. C. D. West, J. Chem. Phys., 17, 219 (1949); S. Saito, H. Okutama, and Y. Fujiyama, Kolloid Z.-Z. Polym., 144, 41 (1955); S. Hayashi and K. Takizawa, Kogyo Kagaku Zasshi, 71, 107 (1968).

4. J. Voelkel and W. Szydlowska, Makromol. Chem., 182, 225 (1981).

5. H. Staudinger, K. Frey, and W. Haehnel, Ber. Dtsch. Chem. Ges., 60, 1782 (1927).

6. S. Hayashi, M. Takayama, and T. Kawamura, Kogyo Kagaku Zasshi, 73, 178 (1970).

7. W. Gallay, Can. J. Research, 14, 105 (1939); L. G. Tebelev and S. A. Glikman, Vysokomol. Soedin., Ser. A; 9, 723 (1967); S. Hayashi, Y. Tanabe, and N. Hojo, Makromol. Chem., 178, 1679 (1977).

8. Y. Tanizaki, T. Kobayashi, and N. Ando, Nippon Kagaku Zasshi, 80, 445 (1959).

9. Y. Oishi and K. Miyasaka, Polym. J., 18, 307 (1986).

10. Y. Kojima, K. Furuhata, and K. Miyasaka, J. Appl. Polym. Sci., 30, 1617 (1985).

11. F. Bueche, "Physical Properties of Polymers," Interscience Publishers, New York, N. Y., 1962, Chapter 13. 